\title{
Online total organic carbon (TOC) monitoring for water and wastewater treatment plants processes and operations optimization
}

\author{
Céline Assmann, Amanda Scott, and Dondra Biller \\ Analytical Instruments, a Division of GE Power, Boulder, Colorado, USA \\ Correspondence to: Céline Assmann (celine.assmann@ge.com) and Amanda Scott (amanda.scott@ge.com) \\ Received: 21 February 2017 - Discussion started: 2 March 2017 \\ Revised: 18 May 2017 - Accepted: 25 May 2017 - Published: 7 August 2017
}

\begin{abstract}
Organic measurements, such as biological oxygen demand (BOD) and chemical oxygen demand (COD) were developed decades ago in order to measure organics in water. Today, these time-consuming measurements are still used as parameters to check the water treatment quality; however, the time required to generate a result, ranging from hours to days, does not allow COD or BOD to be useful process control parameters - see (1) Standard Method 5210 B; 5-day BOD Test, 1997, and (2) ASTM D1252; COD Test, 2012. Online organic carbon monitoring allows for effective process control because results are generated every few minutes. Though it does not replace BOD or COD measurements still required for compliance reporting, it allows for smart, data-driven and rapid decision-making to improve process control and optimization or meet compliances. Thanks to the smart interpretation of generated data and the capability to now take real-time actions, municipal drinking water and wastewater treatment facility operators can positively impact their OPEX (operational expenditure) efficiencies and their capabilities to meet regulatory requirements. This paper describes how three municipal wastewater and drinking water plants gained process insights, and determined optimization opportunities thanks to the implementation of online total organic carbon (TOC) monitoring.
\end{abstract}

\section{Introduction}

Growing populations and expanding industries are pulling on water resources while adding nutrients and pollutants to water sources. These facts coupled with heightened public demand for quality water at affordable prices has the water industry under scrutiny. Whether complying with water regulations, optimizing treatment processes for saving time and money, or looking to better manage a plant during times of emergency (flood, fire, security threat, drought or industrial spill), knowing and understanding organics and organic removal can be extremely valuable. Total organic carbon (TOC) monitoring is one of the most important parameters that drinking water and wastewater facilities can use to make decisions about treatment.

Measuring TOC can be critical to a water treatment facility's water quality in helping to optimize treatment processes. TOC is useful in detecting the presence of many organic con- taminants including petroleum products, organic acids like humic and fulvic acids, pesticides, pathogens, etc. It is a non-specific, but inclusive parameter for monitoring organics. Knowing and understanding TOC levels coming into, throughout, and leaving a plant can be used as a measure of treatment efficacy and as an indicator of contamination. As opposed to methods like biological oxygen demand (BOD) and chemical oxygen demand (COD), TOC includes all organic compounds and can be achieved in a matter of minutes with instrumentation as opposed to hours or days with reagents in a laboratory.

This paper discusses the three organics measurement methodologies mostly used today (BOD, COD, TOC) and provides examples of three municipal drinking water and wastewater treatment plants that have implemented online TOC monitoring as a tool to make informative and rapid treatment decisions, allowing them to optimize their plants processes and operations: City of Boulder (75th Street) Pub- 
lic Works Wastewater Treatment Facility, Colorado (USA), Twin Oaks Valley Water Treatment Plant in San Marcos, California (USA) and City of Englewood Water Treatment Plant, Colorado (USA).

\section{Discussion of the methods for organics measurements and regulatory frameworks}

\subsection{The methods for organics measurements in water and wastewater}

Since the 1970s, laboratory analytical methods for organics measurements have been developed with the aim to establish the concentration (typically in $\mathrm{mg} \mathrm{L}^{-1}$ or $\mathrm{ppm}$ ) of organics (i.e., carbon-containing) matter to determine the relative "strength" of a water and a wastewater sample. Today there are three common laboratory tests used to determine the gross amount of organic matter: BOD, COD and TOC (total organic carbon). Though these tests measure different things in water, there is overlap in the results, and some correlations could be established (see Kiepper, 2016).

\subsubsection{BOD measurements}

BOD measures the amount of dissolved oxygen needed by aerobic biological organisms to oxidize organic material in a water sample. BOD is commonly expressed as BOD5, miligrams of $\mathrm{O}_{2}$ consumed per liter of sample during 5 days of incubation at $20^{\circ} \mathrm{C}$. It is an indirect measurement of organic quality or pollution in water (see Standard Method 5210 B, 1997).

cBOD (carbonaceous BOD) is a BOD measurement where a nitrification inhibitor is added to the BOD sample, to stop the oxidation of ammonia to nitrate, and measure specifically the organic carbon contribution to oxygen demand.

To ensure proper biological activity during the BOD test, a water sample must be free of chlorine and copper, in $\mathrm{pH}$ range 6.5 to 7.5 , and needs to have adequate microbiological population. Besides this, the BOD test is well known to have a challenging reproducibility from person to person, and generates a result after the 5 days of incubation.

\subsubsection{COD measurements}

COD is a popular alternative and complementary test to BOD, with the major advantage that it only takes few hours to complete, compared to the 5 days for BOD. COD analysis is based on the principle to measure the change in color caused by the chemical oxidation of the sample. The oxidation is achieved by closed reflux of a potassium dichromate in sulfuric acid solution. Similarly to BOD Analysis, it is an indirect measurement of organic quality or pollution in water and is commonly expressed as milligrams of $\mathrm{O}_{2}$ consumed per liter of sample (see ASTM D1252, 2012).
COD analysis uses toxic chemicals and generates hazardous waste, that require proper handling and disposal. Indeed, along with the potassium dichromate in $50 \%$ sulfuric acid solution, pre-prepared COD vials also contain silver sulfate as a catalyst and mercuric sulfate to mitigate chloride interferences.

\subsubsection{TOC measurements}

The TOC test is gaining popularity because it only takes 5-10 min to complete. At the heart of the TOC test is a carbon-analyzing instrument that measures the total organic carbon in a water or wastewater sample. There are different types of analyzers, but all oxidize organic carbon into carbon dioxide $\left(\mathrm{CO}_{2}\right)$ and measure that $\mathrm{CO}_{2}$ generated using a detection method. Oxidation methods include combustion, UV persulfate, and super-critical water oxidation while detection methods include NDIR (non-dispersive infrared) and membrane conductivity (see Potter and Wimsatt, 2005, and ASTM D5173-15, 2015).

COD and BOD are laboratory techniques, whereas TOC can be done in the laboratory (offline measurements) or online (at-line measurements). The value of online analysis is obviously getting real-time data to see process changes and make quick process decisions based on the observed fluctuations. Online TOC analyzers typically require maintenance throughout the year and have consumable parts that need to be changed out. Newer TOC analyzers, however, are designed for ease of use and have minimized maintenance down to once per quarter with calibration every 6-12 months.

The cost of ownership and complexity is more important with TOC than with COD or BOD: TOC test procedures are relatively simple and straight forward, but are specific to the type of carbon-analyzing instrument utilized. Thus, no "typical" TOC procedure exists. The instrument manufacturer's procedures should be followed accurately to achieve the best results.

TOC is a highly sensitive, non-specific measurement of the organics present in a sample. Suspended particulate, colloidal and dissolved organic matter are part of the TOC measurement.

\subsection{Regulatory frameworks}

The COD usual method (DIN 38409-H41) is using chromate and mercury, which are toxic chemicals. For this reason, there is a tendency to look for alternatives to the parameter COD and to promote the use of the parameter TOC or chrome-free COD.

In Europe, the development of TOC as a parameter is being reflected in a number of documents, within the Industrial Emissions Directive 2010/75/EU (Integrated Pollution Prevention and Control), such as ROM (Report On Monitoring of Emissions from IED-Installations), final draft document: "total organic carbon (TOC)/chemical oxygen de- 
mand (COD): in some member states, there is a trend to replace COD by TOC for economic and environmental reasons. The use of chromate and mercury, necessary for the COD determination, can be avoided by determining TOC, which can be measured continuously by online analyzers." (see Joint Research Centre, 2017). Some countries, like Sweden, are looking for alternative technologies (see SWWA, 2017).

In the USA, National Pollutant Discharge Elimination System (NPDES) was established under the administration of the Environmental Protection Agency (EPA). With minimal exceptions, NPDES is the primary program that manages discharge limits or effluent limitations guidelines (ELG) for the release of process effluent or wastewater to public waterways (see NPDES Permit Program Basics, 2016 and State NPDES Program Authority, 2016). The NPDES system allows for "authorized alternatives" to oxygen demand, such as TOC measurement, correlating to oxygen demand, as a means for operators to have faster and more accurate monitoring and process control (see NPDES Permit Program, 2015).

\subsection{Discussion about the determination of the correlation factor}

There are a number of ways to properly determine the correlation factor between TOC and the oxygen demand parameter of choice, BOD5 or COD. The method detailed in the Instrumentation Testing Association (ITA) test report is specific with corresponding statistical analyses; refer to the Implementation Protocol (see Nutt and Tran, 2013).

A treatment facility should work with its state NPDES (or other local authority in other countries, like DREAL in France) administrator to execute a long-term, correlation test and replace BOD or COD with TOC as the primary discharge parameter. National regulatory agencies (e.g., USEPA, state DEPs - Departments of Environmental Protection - in the USA) may have specific requirements on the number of samples and test period (see Nutt and Tran, 2013).

\section{City of Boulder Public Works Wastewater Treatment Facility, Colorado (USA)}

\subsection{Method and objective}

The City of Boulder 75th Street Wastewater Treatment Facility (WWTF), USA, gained insight and determined optimization opportunities through the use of online TOC monitoring implemented since March 2015. In addition, the city is looking to gain approval for long-term BOD : TOC correlations (see Babatola and Xu, 2009) from the State of Colorado in order to replace BOD analysis with TOC analysis, which is a faster, easier, and more accurate method of measuring the organic strength of wastewater (see APHA, AWWA, WEF, 2013).
The City of Boulder's (WWTF) 2008 upgrades marked an important transition from a trickling filter/solids contact process to a modified Ludzack-Ettinger (MLE) biological nutrient removal process. The new activated sludge process has successfully reduced effluent ammonia and nitrate concentrations to levels comfortably below current Colorado Discharge Permit System (CDPS) discharge permit limits. However, effective 1 December 2017, the same permit proposes lower daily maximum ammonia limits and new daily maximum nitrate limits. If the Boulder WWTF's future nitrate limit (17.9 $\mathrm{mg} \mathrm{N} \mathrm{L}^{-1}$ for flows $\geq 20 \mathrm{MGD}$ ) were imposed on effluent nitrate quality from 2011 to 2014, 111 violations would appear, illustrating the future vulnerability of the current WWTF configuration.

On-site testing and process modeling pointed to the same cause of incomplete denitrification: a carbon limitation in the anoxic zones of the WWTF's MLE process (see Sigmon et al., 2014). The use of data from an online TOC Analyzer allowed the City of Boulder WWTF to demonstrate that the diurnal patterns of carbon and nitrogen were offset enough to contribute to the WWTF's carbon limitation. This presents a host of optimization opportunities that were previously overlooked as carbon / nitrogen ratios were considered on a daily basis. The city's Nitrogen Upgrades Project, currently in the construction phase, will address the WWTF's carbon limitation by implementing external carbon addition via the sugary by-product of the beer brewing process from a nearby brewery and acetic acid (see Sigmon et al., 2016).

A TOC Analyzer (GE's InnovOx*) is being used in this study to provide online monitoring of aeration basin influent (ABI) TOC concentrations. The analyzer collects a sample from a continuously pumped stream and uses heated persulfate oxidation chemistry assisted by supercritical water to oxidize organic carbon. During this supercritical water oxidation (SCWO), the Analyzer's reactor is heated to $375^{\circ} \mathrm{C}$ and pressurized to $220 \mathrm{bar}$, which conditions are beyond water's critical point.

\subsection{Results and further investigations}

After implementing online TOC analysis, The City of Boulder WWTF demonstrated that the diurnal patterns of carbon and nitrogen are offset enough to contribute to the WWTF's carbon limitation. Data showed that the peak nitrogen loading of the plant occurs approximately $8 \mathrm{~h}$ before the peak carbon loading. Therefore, the biological denitrification process has its highest carbon requirement (due to the highest nitrogen input) hours before it actually receives its highest carbon input. This disconnect between nutrient loading and nutrient requirement presents a host of optimization opportunities that were previously overlooked since as carbon : nitrogen ratios were originally determined via a daily composite which masked the actual offset in the timing of the peak load.

Figure 1a and $\mathrm{b}$ show the diurnal patterns of ammonia and TOC at the ABI and of nitrate at the secondary clarifier in- 


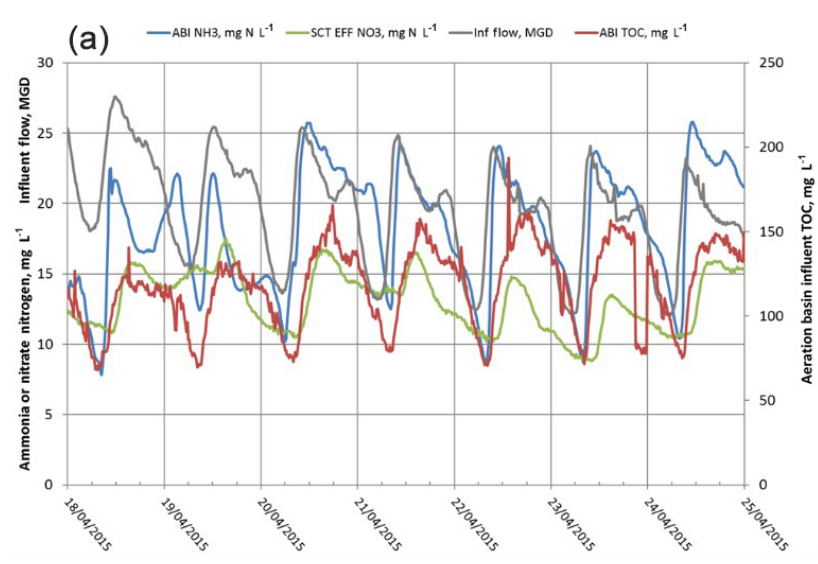

(b) - $\mathrm{ABINH}$, $\mathrm{mg} \mathrm{N} \mathrm{L}^{-1}-\mathrm{SCT}$ EFF NO3, $\mathrm{mg} \mathrm{N} \mathrm{L}^{-1}-\mathrm{ABITOC}, \mathrm{mg} \mathrm{L}^{-1}$

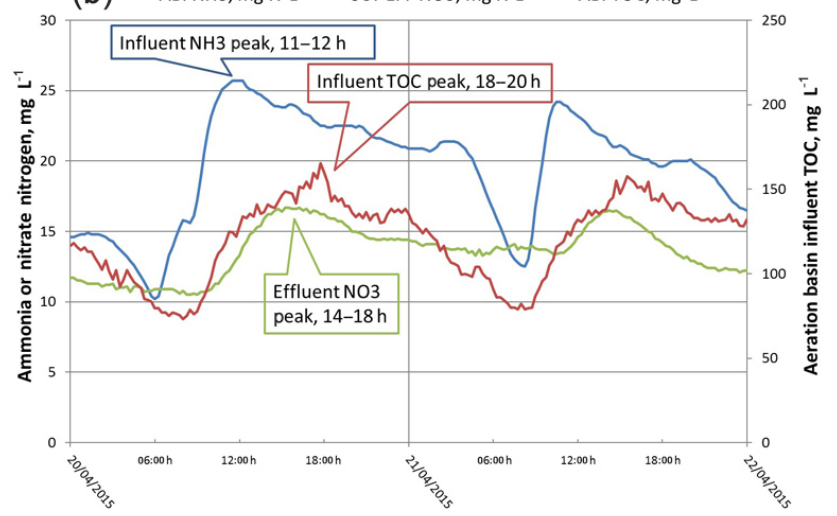

Figure 1. (a) Weekly diurnal patterns of ammonia and TOC at the aeration basin influent $(\mathrm{ABI})$ and of nitrate at the secondary clarifier influent (SCT Eff). (b) Daily diurnal patterns of ammonia and TOC at the ABI and of nitrate at the secondary clarifier influent (SCT Eff).

fluent (SCT Eff) on weekly and daily cycles. Ammonia and nitrate account for the majority of the inorganic nitrogen in the ABI and the SCT Eff, respectively; therefore, these trends can be approximated to be total nitrogen trends on both the influent and effluent of the activated sludge system. As described previously with an $8 \mathrm{~h}$ delay between the daily nitrogen peak, which occurs in the morning (around 11:30LT) and daily carbon peak, which occurs in the early evening (19:00-20:00 LT), it is apparent that nitrogen moves through the activated sludge system before peak influent carbon occurs at the aeration basin influent. This offset in diurnal nitrogen and carbon patterns is a significant contributing factor to the WWTF's carbon limitation.

To further investigate how the offset of diurnal nitrogen and carbon peaks affects denitrification, a calibrated diurnal model will be developed by the plant's engineers using Dynamita's Sumo process simulation software. Key objectives of the modeling effort will be to

- determine the optimum set points for the carbon feed system control philosophy;
- determine how to most efficiently use and control the primary clarifier bypass option;

- adjust the side stream ammonia load to improve the secondary influent $\mathrm{C} / \mathrm{N}$ ratio.

In addition to providing insight into diurnal variability of the WWTF's carbon limitation, TOC is a faster, easier, and more accurate alternative to BOD. Indeed, TOC is a direct measurement of gross amount of organic matter in waters, including suspended particulates, colloidal and dissolved organic matter, while BOD measures the biologically active organic matter indicating amount of oxygen needed for the biological degradation. Every organic compound has a different BOD. Therefore, BOD is poor in precision, and takes 5 days to generates a result, which is not useful for process monitoring. TOC, however, generates a result every few minutes (typically less than 10) and has a more stable baseline.

While BOD and cBOD limits have appeared in NPDES permits since their inception, The Code of Federal Regulations (40CFR133.104(b)), standard methods (APHA, AWWA, WEF, 2013) and the EPA's NPDES Permit Writers' Manual (US EPA, 2010) all allow for the replacement of BOD methods with TOC methods following the development of long-term site-specific correlations (see standard method 5210 B, 1997 and Nutt and Tran, 2013). The City of Boulder's WWTF engaged in a long-term correlation study starting September 2013, measuring TOC in influent, aeration basin influent, and final effluent using several TOC methodologies on $24 \mathrm{~h}$ flow-based composite samples, which were also analyzed for BOD/cBOD. Regression equations were developed from long-term correlations at each process area according to APHA, AWWA, WEF (2013) to estimate BOD and cBOD from TOC and are illustrated in Table 1. These data were submitted to the Colorado Water Quality Control Division for approval and inclusion into the city's CDPS discharge permit, which expired 30 April 2016 and, as of the time of publication, is on administrative extension.

With the number of data pairs used for each correlation, the table shows the linear regression best-fit line equation and $R^{2}$ value associated with each correlation.

\section{Twin Oaks valley Water Treatment Plant in San Marcos, California (USA)}

\subsection{Method and objective}

The Twin Oaks Valley Water Treatment Plant in San Marcos, CA, commissioned in 2008, is a zero discharge plant and one of the world's largest submerged membrane ultrafiltration water treatment plants (100 MGD). The plant uses GE Water \& Process Technologies ZeeWeed $* 1000$ ultrafiltration (UF) membranes in its treatment process. The source water is $95 \%$ surface water that is mixed with reclaim water on-site from an equalization (EQ) basin. The reclaim water is 
Table 1. Summary of the City of Boulder's long-term correlation between BOD and TOC and between cBOD and TOC for both plant influent and final effluent wastewater matrices.

\begin{tabular}{llcll}
\hline $\begin{array}{l}\text { wastewater } \\
\text { matrix }\end{array}$ & Correlation & $\begin{array}{c}\text { Number of } \\
\text { data pairs }\end{array}$ & Linear regression best-fit equation & $R^{2}$ \\
\hline \multirow{2}{*}{ Influent } & BOD : TOC & 27 & BOD $=1.7607($ TOC $)+13.716$ & 0.7123 \\
& cBOD : TOC & 27 & cBOD $=1.2842($ TOC $)+11.184$ & 0.6714 \\
\hline \multirow{2}{*}{ Effluent } & BOD : TOC & 80 & BOD $=1.8464($ TOC $)-8.241$ & 0.5137 \\
& cBOD : TOC & 80 & cBOD $=0.7561($ TOC $)+2.5513$ & 0.3698 \\
\hline
\end{tabular}

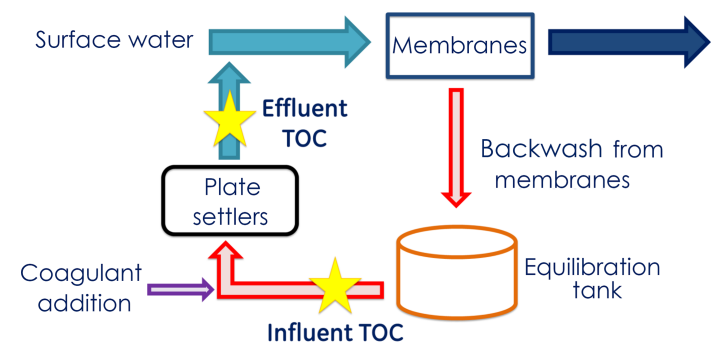

Figure 2. Schematic flow diagram of Twin Oaks Water Treatment Plant's implementation of TOC Analyzer.

primarily backwash from the UF membrane trains. The process of recycling water on-site starts with equalization followed by addition of coagulant/flocculant and then settling in Lamella plate settlers. The settled water is combined with the raw water and fed to the UF membranes. Schematic of the treatment is illustrated in Fig. 2.

In order to optimize membrane performance, treatment processes and organic loading of the membranes must be monitored closely to minimize organic and inorganic fouling potential.

The purpose of this study was to use online TOC monitoring of the influent and effluent to the plate settlers to try to understand why membrane fouling was occurring and then to adjust treatment to prevent fouling from continuing. TOC is used as an analytical tool help understand what is being recycled on-site and how well organics are removed before going back to the membranes. Organics monitoring is important for membrane treatment because organics are the main source of membrane fouling (see Liu, 2017).

Organic carbon levels for the two streams (influent and effluent to the plate settlers) were measured using a Sievers InnovOx Online TOC Analyzer as shown in Fig. 3. The InnovOx Analyzer uses SCWO to oxidize organics and NDIR detection to determine organic carbon concentrations.

For this study, the analyzer was run in non-purgeable organic carbon (NPOC) mode. NPOC mode involves acidification of the sample followed by sparging with $\mathrm{CO}_{2}$-free air in order to remove any inorganic carbon in the sample prior to oxidation.

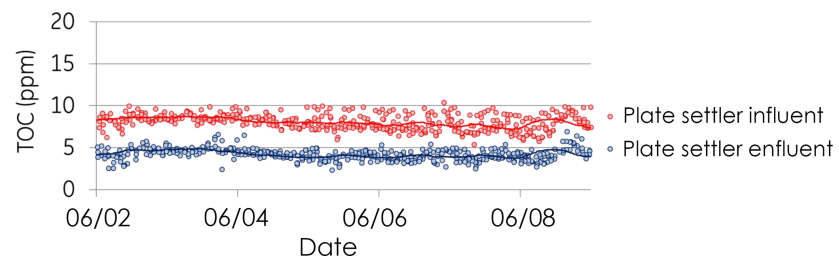

Figure 3. TOC for both streams showing removal from influent to effluent.

\subsection{Results and further investigations}

Near-real-time online analysis of the organic carbon removal for different chemical treatments allowed for rapid understanding of the best treatment options and optimization of treatment as shown in Fig. 2. For example, in this case, online analysis of the organic carbon contributed to understanding source water better and in real-time so smarter decisions could be made to chemical dosages adjustments, protecting membranes from fouling (increasing their life time), and finally contributing to saving money on operational expenditures, while making effluent quality better (see Biller and Mullet, 2016).

Online analysis of plate settler influent and effluent TOC showed an initial TOC removal efficiency of about $40-50 \%$. While trying different chemical treatment options, online TOC analysis provided near-real-time insight into the efficiency of the treatment. Controlling the $\mathrm{pH}$ provided better TOC removal efficiency than adding a different coagulant. This is illustrated in Fig. 4.

Future analysis of online TOC for these two streams will continue to provide information on the organic carbon removal efficiency of reclaim water treatment so that membrane performance can be optimized at this plant. Membrane pre-treatment with $\mathrm{pH}$ control or coagulant changes can help improve membrane lifetimes, increase backwash cycles, and maintain removal efficiency. If pre-treatment is inadequate it can lead to inorganic fouling (too much coagulant) or organic fouling (too much organic material). Thus, proper monitoring of organic removal and chemical usage is key to membrane optimization.

As reclaiming and recycling of water becomes increasingly common at industrial and municipal plants, online 


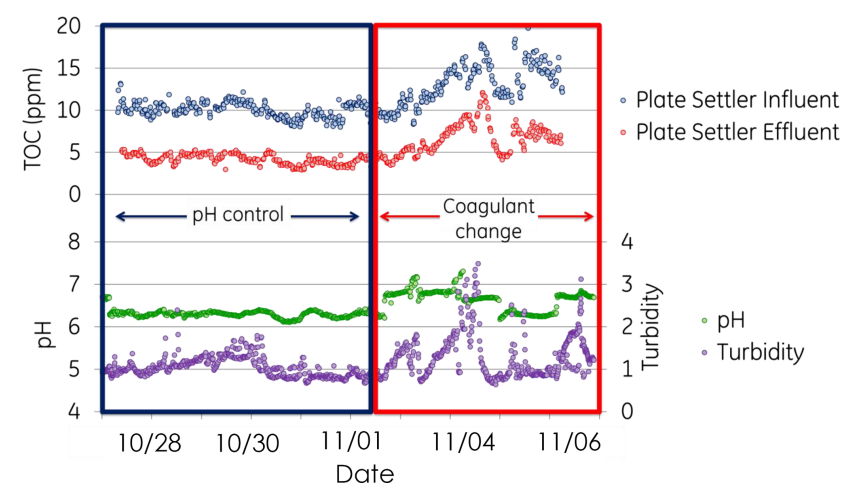

Figure 4. TOC removal efficiencies for $\mathrm{pH}$ control and coagulant changes.

monitoring of TOC should be used so that water treatment can be optimized for maximum TOC removal.

\section{City of Englewood Water Treatment Plant, Colorado (USA)}

\subsection{Objective and method}

One of the most valuable ways that TOC analysis can be used in municipal drinking water plants is to understand the amount of disinfection by-product (DBP) precursors. DBPs form when residual chlorine from disinfection and bromide in water streams react with organic content over time. Known as carcinogens, they are strictly regulated throughout the distribution system. The ultimate dilemma of disinfection is the need to balance disinfectant dosing to control microbial risk with TOC removal to control DBP formation.

Enhanced coagulation is one of the means to decrease TOC content of water. It can be optimized using jar testing as a tool for proactive process control in order to simulate the performance of various chemical coagulants and process conditions without having to test the full-scale treatment process. For many plants, the rule requires optimization of the treatment process to increase the removal of TOC, which can often be improved by selecting the optimum dose of aluminium or ferric-based coagulant. Other treatment parameters including the addition of permanganate, powdered activated carbon, or $\mathrm{pH}$ adjustment can also be easily modeled (see Sytsma et al., 2015).

Traditionally, turbidity and UV254 have been used as primary indicators of good floc formation and removal of organics in jar tests. Turbidity is an indicator of water clarity but does not distinguish between inorganic, organic, or particulate contaminant. UV254 measures the aromatic content of organic matter in water, but not all organic molecules absorb in that wavelength and there are multiple interferences at $254 \mathrm{~nm}$, such as ferric compounds, which can lead to either over or under reporting of the estimated organic carbon content of the water.
More recent testing has shown that TOC may be a far better indicator of a fully optimized treatment process. This is particularly true if TOC measurements can be made immediately as various process changes are made to a jar testing plan. Real-world advantages of fully optimized jar tests may include reduced chemical usage or cost, improved removal of organics, minimization of membrane fouling, minimization of sludge production, and a reduction in regulated DBPs. Jar testing is beneficial for plants so they can optimize their treatment processes to pick the right coagulant type and coagulant dosage.

City of Englewood, $\mathrm{CO}$, is a drinking water treatment plant that treats surface water from South Platte River with a 28 MGD conventional treatment. They were using $60 \mathrm{ppm}$ of coagulant (alum sulfate) and expressed desire to reduce chemical costs.

In order to conduct their process improvement and find cost savings opportunities, the City of Englewood expanded their process data for jar testing from just turbidity to include TOC. Before conducting any trials, they were dosing chemicals blindly to ensure compliance with the new DBP regulations, which require both TOC removal and minimizing formation of DBPs at the furthest point in their distribution system. By dosing excess chemicals, they were able to meet regulations but this also led to high chemical costs, high sludge production and costly sludge removal.

\subsection{Results and further investigation}

They managed to reduce operational cost expenditures within several steps of plant optimization, including the ability to change $\mathrm{pH}$, coagulant type or coagulant dosage to obtain optimum results and ensure removal of organics and know when to regenerate granular activated carbon (GAC).

By having TOC analysis on-site and jar testing data with TOC and turbidity, plant operators did not have to wait for third party test results and could make immediate process decisions.

The plant was able to save over USD $100 \mathrm{k}$ in chemicals and disposal costs and shown in Table 2. They also realized that effective TOC removal does not always correlate to effective turbidity removal or vice versa; therefore, TOC and turbidity levels must both be monitored. Typical coagulants can remove TOC to a certain degree, beyond that amount excess chemical is a waste of money and requires excess sludge removal. Characteristics of a plant's source water can change rapidly, including $\mathrm{pH}$, alkalinity and the organic composition of the water. Online TOC monitoring is the most effective means for frequent process observation.

Further investigation consists in using TOC data and TOC characterization to try and better understand what types of organics are impacting treatment such as coagulant dose, DBP formation and membrane fouling. Also, a better understanding of source water characteristics and organic loading can help size system processes. As water reuse systems be- 
Table 2. Chemical and disposal cost savings achieved by adding in TOC analysis.

\begin{tabular}{|c|c|c|c|c|c|c|c|}
\hline & $\begin{array}{c}\text { Dosage } \\
\left(\mathrm{mg} \mathrm{L}^{-1}\right)\end{array}$ & $\begin{array}{l}\text { Coagulant } \\
\text { usage/year }\end{array}$ & $\begin{array}{l}\text { Coagulant } \\
\text { costs/year }\end{array}$ & $\begin{array}{l}\text { Coagulant } \\
\text { savings/ } \\
\text { year }\end{array}$ & $\begin{array}{l}\text { Coagulant } \\
\text { waste/year }\end{array}$ & $\begin{array}{l}\text { Disposal } \\
\text { costs/year }\end{array}$ & $\begin{array}{l}\text { Total } \\
\text { savings/ } \\
\text { year }\end{array}$ \\
\hline Stage 1: D/DBPR implemented & 60 & $1410588 \mathrm{lbs}$ & USD 136827 & NA & 1830 yards $^{3}$ & USD 100650 & NA \\
\hline Coagulant reduction & 45 & $959049 \mathrm{lbs}$ & USD 106454 & USD 30373 & 1250 yards $^{3}$ & USD 68750 & USD 62723 \\
\hline 1st optimization study with TOC & 36 & $728028 \mathrm{lbs}$ & USD 86003 & USD 50824 & 920 yards $^{3}$ & USD 50600 & USD 100874 \\
\hline 2nd optimization study with TOC & 20 & $426174^{*}$ & USD $53698^{*}$ & USD $83129 *$ & 700 yards $^{3, *}$ & USD $38500^{*}$ & USD $145279^{*}$ \\
\hline
\end{tabular}

* Usage, costs and savings are calculated for 1 year based on current dosage rate recently implemented. NA = not available.

come more viable, TOC analysis gains interest as an indicator for the health of each train in a multiple barrier treatment process, helping both to protect human and environmental health.

\section{Conclusions}

Online organic carbon monitoring drives smart, informative and rapid decision-making to improve process control of drinking water and wastewater treatment plants so that these treatment facilities can meet regulatory compliances and/or optimize treatment process. Municipal treatment facility operators can use data to make real-time actions that impact their OPEX (operational expenditure) spending and their capabilities to meet regulatory requirements.

These three examples of plants demonstrated that the use of data from a TOC analyzer provides insights of realtime variations of organic carbon, that can be used to optimize processes, ranging from nutrient dosing at a biological wastewater treatment facility to treating membrane backwash water to minimizing DBP formation potential in drinking water.

Implementing TOC analysis at water treatment facilities is a powerful tool that can help operators continue to effectively treat water and positively impact the costs of treatment, in order to meet current and future regulatory requirements.

Data availability. Readers can access the underlying research data by contacting the authors quoted as references for the availability of the data sets.

The Supplement related to this article is available online at https://doi.org/10.5194/dwes-10-61-2017-supplement.

Competing interests. The authors declare that they have no conflict of interest.

Special issue statement. This article is part of the special issue "Computing and Control for the Water Industry, CCWI 2016". It is a result of the 14th International CCWI Conference, Amsterdam, the Netherlands, 7-9 November 2016.

Edited by: Edo Abraham

Reviewed by: two anonymous referees

\section{References}

APHA, AWWA, WEF: Standard Methods for Examination of Water and Wastewater, 22nd Edn., American Public Health Association, Washington, 1360 pp., 2013.

ASTM D1252-06(2012)e1: Standard Test Methods for Chemical Oxygen Demand (Dichromate Oxygen Demand) of Water, ASTM International, West Conshohocken, PA, available at: http: //www.astm.org (last access: 23 June 2017, 2012.

ASTM D5173-15: Standard Guide for On-Line Monitoring of Total Organic Carbon in Water by Oxidation and Detection of Resulting Carbon Dioxide, ASTM International, West Conshohocken, PA, 2015.

Babatola, A. and Xu, T.: Laboratory Development of Site-specific Equations for BOD to TOC Conversion and Application in NPDES and Treatment Process Control, WEFTEC 2009, Orlando, FL, 2009.

Biller, D. and Mullet, M.: Optimizing Treatment of Reclaimed Water at a Drinking Water Plant by Online Monitoring of Organic Carbon Levels, Pittcon, Atlanta, GA, 2016.

Joint Research Centre Directorate Growth and Innovation Circular Economy and Industrial Leadership Unit European IPPC Bureau: JRC Reference Report on Monitoring of Emissions to Air and Water from IED installations, Sevilla, Spain, p. 100, 2017.

Kiepper, B.: Of the University of Georgia Biological \& Agricultural Engineering and Poultry Science Departments, and the Faculty of Engineering Outreach Service: Understanding Laboratory Wastewater Tests: ORGANICS (BOD, COD, TOC, O \& G), Athens, GA, 2016.

Liu, C.: Flexible Cleaning Regimes Promote Membrane Permeability, AWWA OPFLOW, 43, 28-30, 2017.

NPDES Permit Program: Central Tenets of the National Pollutant Discharge Elimination System (NPDES) Permitting Program: Page 2. United States Environmental Protection Agency, http:// water.epa.gov/polwaste/npdes/basics/upload/tenets.pdf, last updated: 7 April 2015.

NPDES Permit Program Basics: United States Environmental Protection Agency, https://www.epa.gov/npdes/ npdes-permit-basics, last access: 24 August 2016.

Nutt, S. G. and Tran, J.: of XCG Consultants Ltd.: Addressing BOD5 limitations through Total Organic Carbon Correlations: A 
Five Facility International Investigation, Pensacola, Florida: water \& Wastewater Instrumentation Testing Association of North America (ITA), Pensacola, Florida, January 2013.

Potter, B. B. and Wimsatt, J. C.: Method 415.3 Determination of total carbon and specific UV absorbance at $254 \mathrm{~nm}$ in source water and drinking water, available at: https://cfpub.epa.gov/si/si_ public_file_download.cfm?p_download_id=525079 (last access: 21 July 2017), 2005.

Sigmon, C., Weirich, S., and Douville, C.: The Best Carbon for the Job: Using the 2010 WERF Protocol to Choose an External Carbon Alternative for Enhanced Nitrate Removal, WEFTEC 2014, New Orleans, LA, 2014.

Sigmon, C., Mimna, M., Santiago, L., and Mullet, M.: TOC Talks: Insight and Efficiency at the City of Boulder's Wastewater Treatment Facility, WEFTEC 2016, New Orleans, LA, 2016.
Standard Method 5210 B (5-day BOD Test): Biochemical Oxygan Demand (BOD), United States Environmental Protection Agency, 1997, available at: https://www.epa.gov/ (last access: 23 June 2017), 1997.

State NPDES Program Authority: United States Environmental Protection Agency, https://www.epa.gov/npdes/ npdes-state-program-information, last updated: 19 February 2016.

SWWA: Swedish Wastewater Association Research \& Development, available at: http://www.svensktvatten.se, last access: 23 June 2017.

Sytsma, S., Scott, A., and Biller, D.: Improved Jar Testing Optimization with TOC Analysis, CA-NV AWWA Fall 2015, Las Vegas, NV, 2015.

US EPA: NPDES Permit Writer's Manual, EPA, Washington, D.C., 2010. 